\title{
A Fast Method of Deriving the Kirchhoff Formula for Moving Surfaces
}

\section{F. Farassat and Joe W. Posey NASA Langley Research Center Hampton, Virginia}

Presented at the 154th Meeting of the Acoustical Society of America 27 November- 1 December 2007, New Orleans, Louisiana 


\section{Outline}

- Motivation and the Problem

- The Imbedding Method

- Generalized Differentiation Rules

- Classical Kirchhoff Formula

- Kirchhoff Formula for a Moving Surface

- Concluding Remarks 


\section{Motivation and the Problem} Helicopter rotor noise prediction

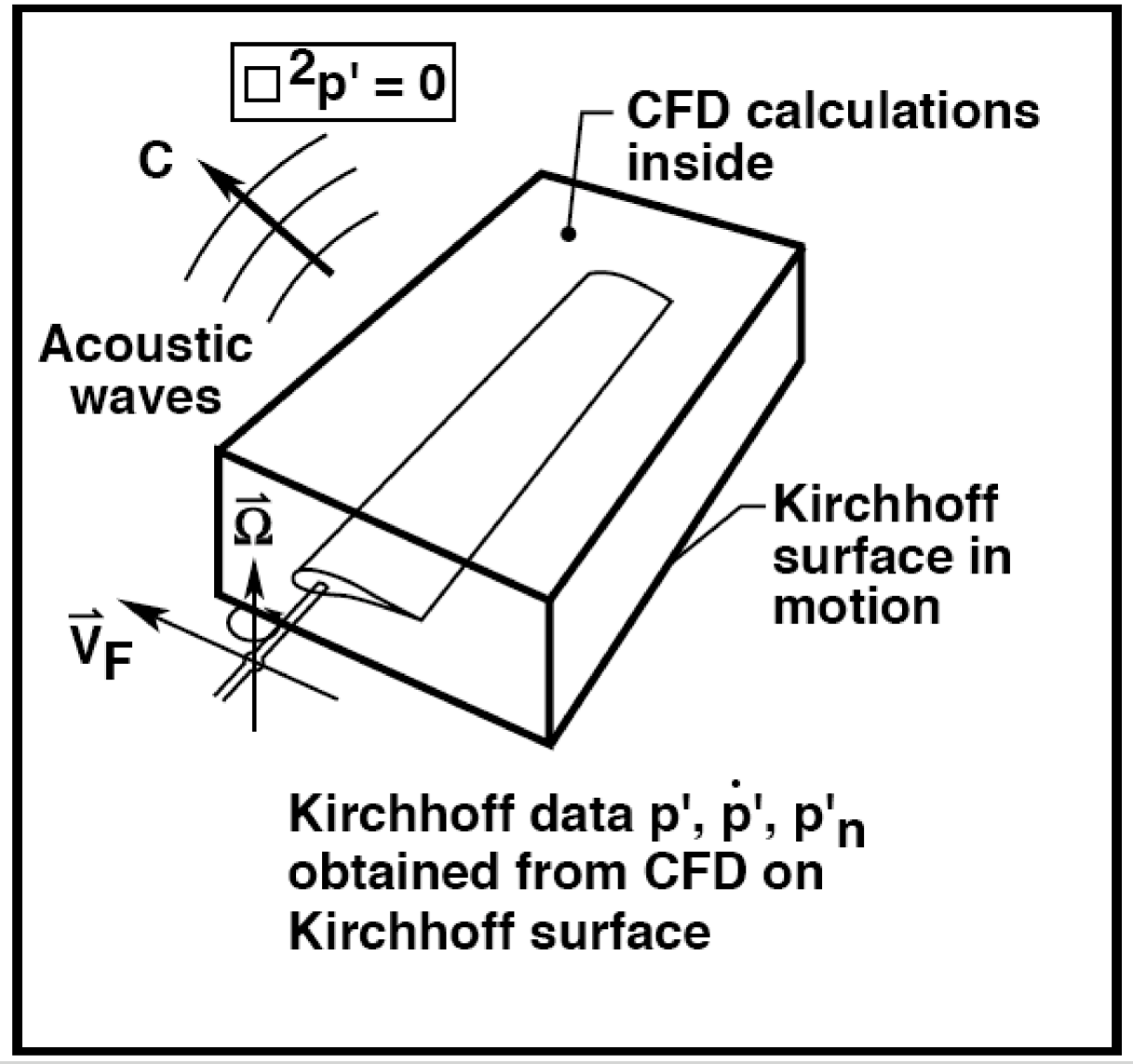

Kirchhoff formula for a moving surface gives $\mathbf{p}$ ' in the exterior region from surface data. 


\section{Derivation of Kirchhoff Formula for a Moving Surface}

\section{Classical Analysis}

- 4D Green's Theorem required

- Some ambiguities working with 4D integrals

- Extremely involved algebraic manipulations

- Difficulties with physical meaning of some terms

\section{References:}

Classical analysis: W. R. Morgans: The Kirchhoff formula extended to a moving surface, Philosophical Magazine, 9, 1930, 141-161

Modern analysis using generalized functions: F. Farassat and M. K. Myers: Extension of Kirchhoff's formula to radiation from moving sources, Journal of Sound and Vibration, 123 (3), 1988, 451-460 


\section{| The Imbedding Method- The Idea}

- When the Green's function $G(x, y)$ of a differential equation $\mathcal{L} u=$ $f$ is known in the a domain $\Sigma$, then that Green's function can be utilized in solving the same differential equation in a smaller domain $\Sigma^{\prime} \subset \Sigma$. 


\section{| The Imbedding Method- The Idea}

- When the Green's function $G(x, y)$ of a differential equation $\mathcal{L} u=$ $f$ is known in the a domain $\Sigma$, then that Green's function can be utilized in solving the same differential equation in a smaller domain $\Sigma^{\prime} \subset \Sigma$.

- The problem in the domain $\Sigma^{\prime}$ is imbedded in the larger domain $\Sigma$ by giving a known value to the unknown function in the region $\Sigma \backslash \Sigma^{\prime}$ which satisfies the boundary conditions of the problem in $\Sigma$. 


\section{| The Imbedding Method- The Idea}

- When the Green's function $G(x, y)$ of a differential equation $\mathcal{L} u=$ $f$ is known in the a domain $\Sigma$, then that Green's function can be utilized in solving the same differential equation in a smaller domain $\Sigma^{\prime} \subset \Sigma$.

- The problem in the domain $\Sigma^{\prime}$ is imbedded in the larger domain $\Sigma$ by giving a known value to the unknown function in the region $\Sigma \backslash \Sigma^{\prime}$ which satisfies the boundary conditions of the problem in $\Sigma$.

- The imbedded problem, in general, has a discontinuous solution at the boundary surface $\partial \Sigma^{\prime}$ of the domain $\Sigma^{\prime}$. This means that the best tool to solve the imbedded problem is the generalized function (GF) theory. 


\section{The Imbedding Method (cont'd)}

- The Green's function method is applicable in finding the discontinuous solution of differential equation as long as all the derivatives are viewed as generalized derivatives.

\section{References:}

F. Farassat, Introduction to Generalized Functions With Applications in Aerodynamics and Acoustics, NASA Technical Paper 3428, 1994

F. Farassat: The Kirchhoff Formulas for Moving Surfaces in Aeroacoustics The Subsonic and Supersonic Cases, NASA Technical Memorandum 110285, September 1996

F. Farassat, et. al: Working With the Wave Equation in Aeroacoustics- The Pleasures of Generalized Functions, AIAA-2007-3562, 2007 


\section{An Example of the Imbedding Method}

Consider $l u(x)=u^{\prime \prime}$ on $[0,1], \quad$ BCs: $u(0)-2 u^{\prime}(0)=0 \quad \&$ $u(1)+u^{\prime}(\mathbf{1})=0$. The Green's function is

$$
g(x, y)= \begin{cases}\frac{(y-2)(x+2)}{4} & x<y \\ \frac{(y+2)(x-2)}{4} & x>y\end{cases}
$$

Use above Green's function to solve the same ODE $l u(x)=u^{\prime \prime}(x)=k(x)$ on $[\mathbf{a}, \mathbf{b}] \subset[0,1]$ by the imbedding method. Let unknown function take value 0 on $[0,1] \backslash[a, b]$.

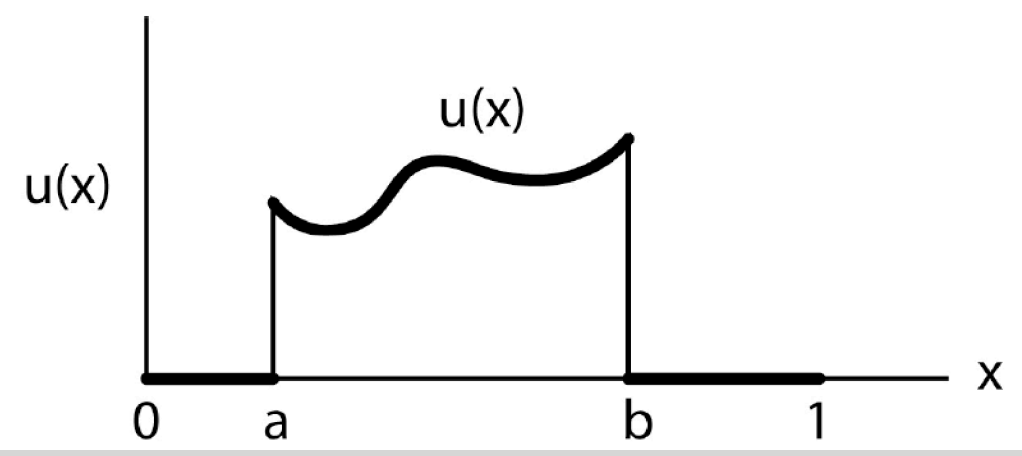

Then use generalized function (GF) theory to find new ODE. 


\section{Generalized Differentiation Rules}

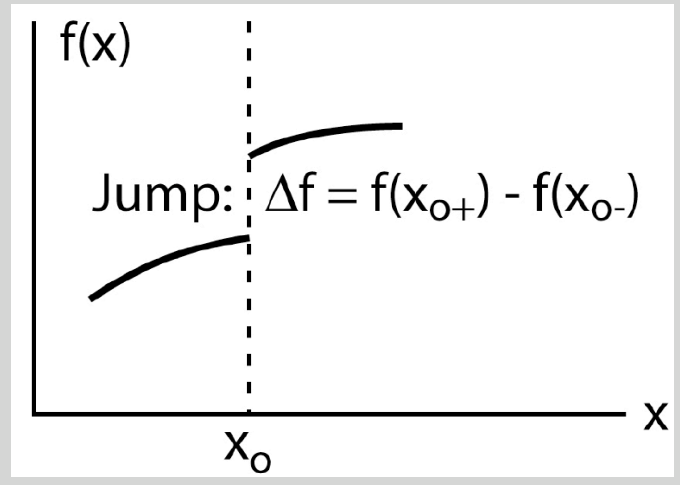

Generalized derivative (GD) of a differentiable function with a single jump discontinuity at $x_{0}$ is

$$
\bar{f}^{\prime}(x)=f^{\prime}(x)+\triangle f \delta\left(x-x_{0}\right)
$$

GD of a function $\mathbf{k}(\mathbf{x})$ in multidimensions with a discontinuity $\Delta k$ across a surface $f=0$, $\nabla f=n$, is

$$
\bar{\nabla} k(\boldsymbol{x})=\nabla k(x)+\Delta k \boldsymbol{n} \delta(f)
$$


An Example of the Imbedding Method (cont'd) New ODE of example imbedded problem is:

$$
\begin{aligned}
\overline{\tilde{u}}^{\prime \prime}(x)= & \tilde{k}(x)+u^{\prime}(a) \delta(x-a)-u^{\prime}(b) \delta(x-b) \\
& +u(a) \delta^{\prime}(x-a)-u(b) \delta^{\prime}(x-b)
\end{aligned}
$$

with the following definitions:

$$
\begin{aligned}
& \tilde{u}(x)=\left\{\begin{array}{lr}
u(x) & x \in[a, b] \\
0 & x \varepsilon[0,1] \backslash[a, b]
\end{array}\right. \\
& \tilde{k}(x)=\left\{\begin{array}{lr}
k(x) & x \in[a, b] \\
0 & x \varepsilon[0,1] \backslash[a, b]
\end{array}\right.
\end{aligned}
$$




\section{Classical Kirchhoff Formula}

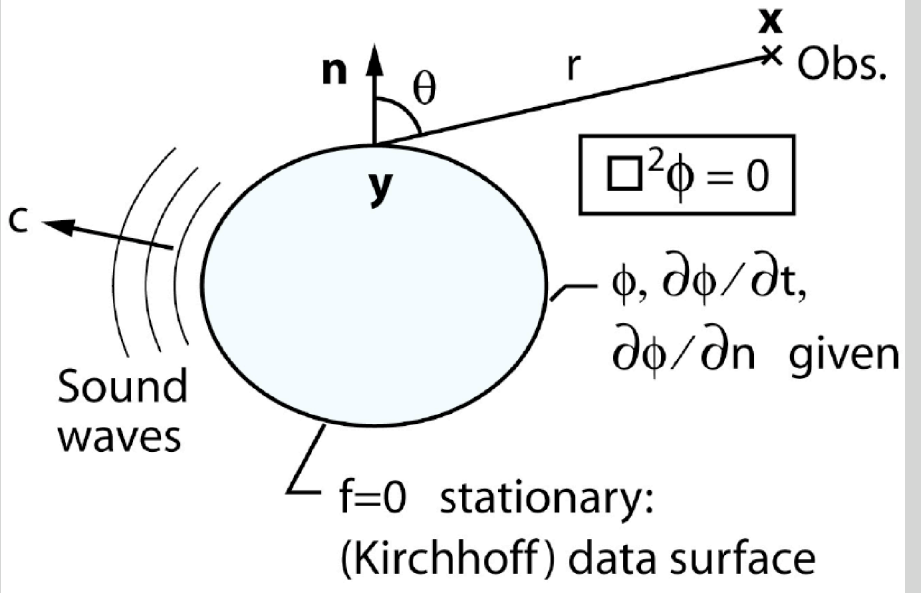

Imbed this problem in unbounded 3D space by assuming that the unknown function is 0 inside the surface $\mathbf{f}=\mathbf{0}(\nabla \mathbf{f}=\mathbf{n})$. Let

$$
\tilde{\varphi}(\boldsymbol{x}, t)= \begin{cases}\varphi(\boldsymbol{x}, t) & f>0 \\ 0 & f<0\end{cases}
$$




\section{Classical Kirchhoff Formula (cont'd)}

New PDE to derive the classical Kirchhoff formula is:

$$
\bar{\square}^{2} \tilde{\varphi}=-\varphi_{n} \delta(f)-\nabla \cdot[\varphi \boldsymbol{n} \delta(f)]
$$

Next use the Green's function of the wave equation in the unbounded space to get the result.

\section{The rest is simple algebraic manipulations!}




\section{Kirchhoff Formula for a Moving Surface}

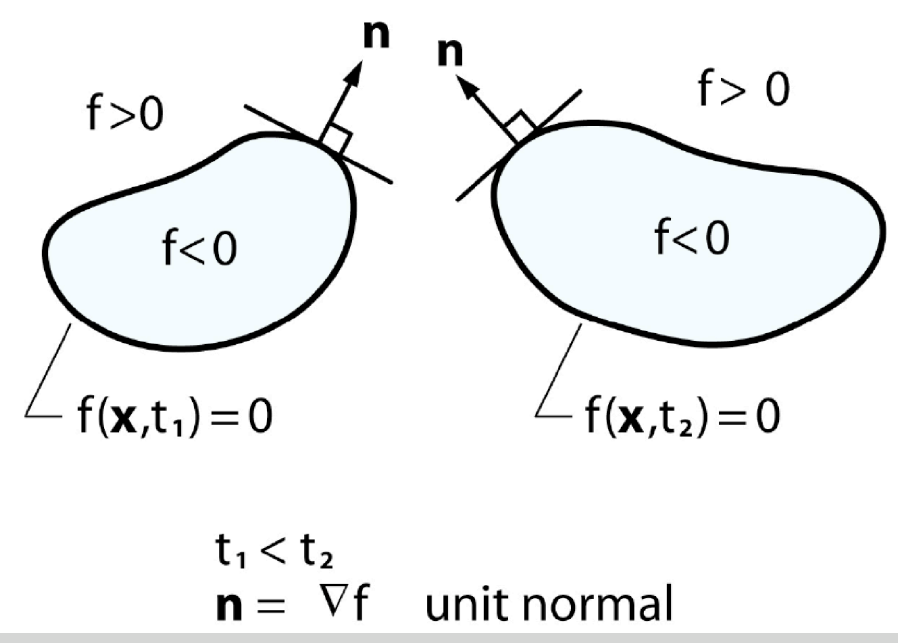

To solve $\square^{2} \varphi=Q(x, t)$ in the exterior of the moving surface $f=0$, imbed this problem in unbounded 3D space taking the value 0 inside the moving surface. Let:

$$
\begin{array}{r}
\tilde{\varphi}(\boldsymbol{x}, t)= \begin{cases}\varphi(\boldsymbol{x}, t) & f>0 \\
0 & f<0\end{cases} \\
\tilde{Q}(\boldsymbol{x}, t)= \begin{cases}Q(\boldsymbol{x}, t) & f>0 \\
0 & f<0\end{cases}
\end{array}
$$


Kirchhoff Formula for a Moving Surface (cont'd) New PDE to derive Kirchhoff formula for a moving surface is:

$$
\begin{aligned}
& \bar{\square}^{2} \tilde{\varphi}=\tilde{Q}(\boldsymbol{x}, t)-\frac{1}{c} \varphi_{t} M_{n} \delta(f) \\
& -\frac{1}{c} \frac{\partial}{\partial t}\left[\varphi M_{n} \delta(f)\right]-\varphi_{n} \delta(f)-\nabla \cdot[\varphi \boldsymbol{n} \delta(f)]
\end{aligned}
$$

Use the Green's function of the wave equation in the unbounded space to get the result.

The rest is again simple algebraic manipulations! 


\section{Concluding Remarks}

The imbedding method increases the utility of a known Green's function in solving new BV and IV problems of acoustics and other applied fields.

\section{Generalized function theory provides}

a fast method of finding both the

classical Kirchhoff formula and the

Kirchhoff formula for a moving surface. 